\title{
Internal Electrical Noises of BioFET Sensors Based on Various Architectures
}

\author{
Lusine Gasparyan ${ }^{1,2}$, Ferdinand Gasparyan1, Vahan Simonyan ${ }^{2}$ \\ ${ }^{1}$ Yerevan State University, Yerevan, Armenia \\ ${ }^{2}$ DNA-HIVE LLC, Rockville, MD, USA \\ Email: fgaspar@ysu.am
}

How to cite this paper: Gasparyan, L., Gasparyan, F. and Simonyan, V. (2021) Internal Electrical Noises of BioFET Sensors Based on Various Architectures. Open Journal of Biophysics, 11, 177-204. https://doi.org/10.4236/ojbiphy.2021.112006

Received: February 1, 2021

Accepted: March 26, 2021

Published: March 29, 2021

Copyright $\odot 2021$ by author(s) and Scientific Research Publishing Inc. This work is licensed under the Creative Commons Attribution International License (CC BY 4.0)

http://creativecommons.org/licenses/by/4.0/ (c) (i) Open Access

\begin{abstract}
The results of a comparative literature analysis of internal electrical noises and signal-to-noise ratio for nanoscale BioFET (biological field-effect transistor) and DNA (deoxyribonucleic acid) sensors based on different architectures MIS (metal-insulator-semiconductor), EIS (electrolyte-insulator-semiconductor) and ISFET (ion-selective field-effect transistor) are presented. Main types, models and mechanisms of internal noises of bio- \& chemical field-effect based sensors are analyzed, summarized and presented. For the first time, corresponding detail electrical equivalent circuits were built to calculate the spectral densities of noises generated in the active part of a solid (semiconductor, dielectric) and in an aqueous solution for MIS, EIS and ISFET structures based sensors. Complete expressions are obtained for the rms (root mean square) value of the noise current (or voltage), as well as the noise spectral densities for the architectures under study. The miniaturization of biosensors leads to a decrease in the level of the useful signal-current. For successful operation of the sensor, it is necessary to ensure a high value of the SNR (signal-to-noise ratio). In case of weak useful signals, it is necessary to reduce the level of internal electrical noise. This work is devoted to a detailed study of the types and mechanisms of internal electrical noises in specific biosensor architectures.
\end{abstract}

\section{Keywords}

Noise, Sensor, BioFET, Signal-to-Noise Ratio

\section{Introduction}

The field-effect transistor-based biosensors (BioFETs) [ion sensitive field effect transistors (ISFET), electrolyte-insulator-semiconductor (EIS) structures, its 
modifications] are a potential candidate for future bioassay applications due to its low cost, fast response, high sensitivity and small sensing size. The $\mathrm{pH}$-sensitive ISFETs are very important sensors for in vivo continuous monitoring application of physiological and environmental system. The accuracy of ISFET output measurement is greatly affected by the presences of internal noise, drift and slow response of the device. Although the noise analysis of BioFETs so far performed in different literature relates only to sources originated from FET structure which is almost constant for a particular device, the pH-dependent electrochemical noise has not been substantially explored and analyzed in detail. Reliable ways of DNA sequencing by ionic and tunneling current require low-noise and high-speed measurements of current in aqueous environments [1] [2] [3]. In nanopore experiments that involve ionic and/or tunnel current detection, several sources of noise need to be recognized and reduced. In addition, when a voltage is applied across the nanopore, a steep increase in low-frequency (LF) noise is observed. This region of the noise spectrum is called $1 / f$-noise, which is due to transient factors that influence the current flux (e.g., surface charge fluctuations, hydrophobic pockets on the pore surface, etc.) [4] [5] [6] [7]. Fluctuations of the environment parameters, such as ions and DNA molecules motion, introduce important scattering processes that may affect the viability of this approach to sequencing. A simple model that captures the role of this complex environment in electronic dephasing and its ability to remove charge carriers from current-carrying states is analyzed in [8]. It is finding that these effects do not strongly influence the current distributions due to the off-resonant nature of tunneling through the nucleotides. This result is expected to be a common feature of transport in molecular compounds. In particular, only large scattering strengths, as compared to the energetic gap between the molecular states and the Fermi level, significantly alter the form of the current distributions. Since this gap itself is quite large, the current distributions remain protected from this type of noise, further supporting the possibility of using transverse electronic transport measurements for DNA sequencing.

Previous works showed the four DNA nucleotides possess statistically distinguishable electronic signatures [9] [10] in the form of current distributions when accounting for structural distortions and partial control of the DNA dynamics (i.e., by a transverse field) [2] [9] [10] [11] [12] [13]. These results indicate DNA sequencing is, in principle, possible via transverse current measurements. However, such studies have neglected scattering processes, such as fluctuations of the environment, which introduce current noise, and may thus affect the ability to distinguish the bases. It is clear that the noise of the force signal connected with the orientation of the bases in the pore on the passing current will be added, and the current signal will be distorted. The environment is composed of ionic and water fluctuations and other excitations that may drastically affect the electron dynamics, and thus the current and noise [14]. The complexity of the problem considered by Krems with coauthors, both in the number of atoms involved and 
the type of scattering processes to take into account [8].

A solid-state nanopore platform with a low noise level and sufficient sensitivity to discriminate ssDNA homopolymers of poly-A40 and poly-T40 using ionic current blockade sensing are proposed and demonstrated in [15]. The key features of this platform are:

1) highly insulating dielectric substrates that are used to mitigate the effect of parasitic capacitance elements, which decrease the ionic current root mean square (rms) noise level to sub-10 pA and

2) ultra-thin silicon nitride membranes with a physical thickness of $5 \mathrm{~nm}$ (an effective thickness of $2.4 \mathrm{~nm}$ estimated from the ionic current) are used to maximize the SNR and the spatial depth resolution.

The reliable formation of small nanopores ( $<2 \mathrm{~nm}$ in diameter), fabrication of an extremely thin sensing zone with a thickness comparable to the spacing of each nucleotide, decrease of the noise level, and control of the translocation speed that would guarantee sufficient time to sense each nucleotide are the few challenges that limit the performance of solid-state nanopores. Among these issues, the excess noise level in solid-state nanopores (a few tens of pA to $100 \mathrm{pA}$, 10 times larger than that of protein counterparts [17] [18] [19] [20]) has been one of the key issues responsible for the degraded signal-to-noise ratio (SNR) and temporal and spatial resolution. In particular, the elevated parasitic capacitance generates a high level of dielectric noise that prevents sampling at high bandwidths. A novel solid-state nanopore platform with a sub-10 pA noise level by fabricating a $\mathrm{SiN}_{\mathrm{x}}$ membrane directly on top of highly dielectric substrates proposed in [15]. It is demonstrated that high-frequency noise signals can be significantly reduced by replacing the commonly used Si substrate with an insulating one. Nanopore fabrication method presented in [16] consistently produces nanopores with low $1 / f$-noise levels (see Figure 1 ), comparable to fully wetted transmission electron microscopy (TEM)-drilled nanopores.

In [21] noise was calculated from the current versus time curves recorded in the solvent at $50 \mathrm{kHz}$ sampling rates with $100 \mathrm{kHz}$ low-pass filtering. The noise spectra showed linear components at the frequency above $10^{2} \mathrm{~Hz}$. This feature is

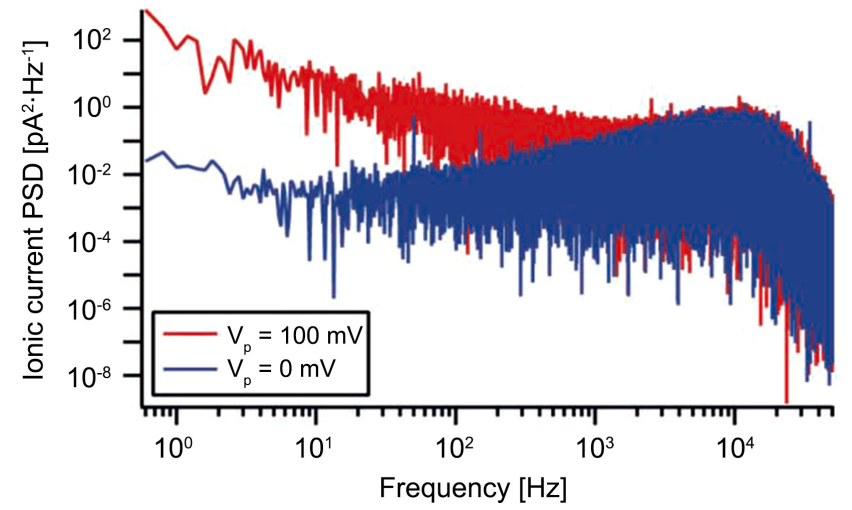

Figure 1. Power spectra density (PSD) of the ionic current at pore voltage of 0 and 100 $\mathrm{mV}$. Root mean square noise of $40 \mathrm{pA}$ is usually achieved at $\mathrm{Vp}=0$. Adapted from [16]. 
naturally interpreted as stemming from the voltage noise in the current amplifier coupled to the net capacitance of the mechanically controllable break junction (MCBJ) system. Morikawa reported also a use of insulator-protected nanoprobes for achieving $7.6 \mathrm{pA} \mathrm{rms}$ noise at $50 \mathrm{kHz}$ sampling rates in an electrolyte solution [22]. Dielectric-covered MCBJ to create a pair of nano-exposed insulator/Au coaxial electrodes closely separated by $1 \mathrm{~nm}$ were utilized. It is observed insulator layer thickness dependent noise levels, which was attributed to decreased capacitance through replacing capacitive electric double layers by the low-k dielectrics.

The LF $\mathrm{pH}$-dependent electrochemical noises that originate from the ionic conductance of the electrode-electrolyte-FET structure of the device and that the noise depends on the concentration of the electrolyte and $1 / f$ in nature are investigated in [23]. The statistical and frequency analysis of this electrochemical noise of a commercial ISFET sensor, under room temperature has been performed for six different $\mathrm{pH}$ values ranging from $\mathrm{pH} 2$ to $\mathrm{pH} 9.2$. It is also proposed a concentration dependent $a / f$ and $b / f^{2}$ model of the noise with different values of the coefficients $a$ and $b$.

In [24] the small-signal and noise modeling of BioFET sensors implemented with EIS structures is studied, with emphasis on design guidelines for low-noise performance. In doing so, a modified form of the general charge sheet MOSFET model that better fits the EIS structure is used. It is discussed how if the reference electrode and the insulator-electrolyte generate no noise associated with charge transport, then the main noise mechanisms are the resistive losses of the electrolyte and the LF noise of the FET. It is found that for realistic sensor geometries and high electrolyte concentrations, the noise from the FET dominates the thermal noise from the electrolyte resistance, and the optimal biasing point for the FET for minimum noise is found to be around moderate inversion.

Syu with coauthors discusses how BioFET sensor can be designed by CMOS platform and the integration with sample processing and data processing apparatus for clinical sample testing [25]. This technology by clinical application is categorized, and a brief summary and comparison for BioFET sensor solutions are provided to each clinical problem. Because most of biomolecules carry electrostatic charges and bioactivities involve electrical potential changes. Noise contribution of commercial ISFETs, as well as of new designed pH-sensors, is studied and measured. Selected CMOS technology offers lower noise properties for designed ISFETs compared to commercial device, which is an advantage.

With the increased use of ISFET as a commercial micro- and nano-BioFET sensor for accurate biomedical measurements, noise factors will determine the performance limits of the system. The total noise comes from the ISFET sensor itself, as well as from the read-out circuit. The important noise components are present in a MOS transistor. The first one is the thermal noise, and the other 1/ $f$-noise.

The study of noise in BioFETs is important for the reason that any source of 
noise present in the sensor will impose a fundamental limit on the resolution and accuracy of the measurements and, hence, sensitivity of BioFETs limited by the sensor noise. In BioFET sensors presents several intrinsic and extrinsic rms noise sources [26]:

1) Intrinsic noise $\overline{i_{d 1}^{2}}$ generated by the electronic device itself ( $1 / f$-noise, channel thermal noise, source and drain diffusion resistance thermal noises, substrate resistance noise, shot noise).

2) Extrinsic electrochemical noise $\overline{i_{d 2}^{2}}$ generated by ion-membrane interactions, in the solution and at the reference electrode.

3) Extrinsic noise source $\overline{i_{d 3}^{2}}$ coming from biasing elements (power supplies, reference electrode biasing, external biasing resistances). These noise sources can generally be filtered.

The BioFET total noise spectrum can be presented as

$$
\overline{i_{n d}^{2}}=\overline{i_{d 1}^{2}}+\overline{i_{d 2}^{2}} \text {. }
$$

Extrinsic electrochemical noise has been modeled in [27]. Another noise analytical model was introduced in [28] based on site-binding model [29] electrochemical equilibrium equations and kinetic reaction rate expressions. The model described charge distributions in the electrolyte-oxide interfacial region and the manner in which ions are transferred between them.

The trend towards miniaturization of biosensors leads to a decrease in the level of the useful signal current. A high signal-to-noise ratio must be ensured for the sensor to work successfully. In case of weak wanted signals, it is necessary to reduce the level of internal electrical noise. As follows from the analysis of literature data, many types of internal electrical noises with different generation mechanisms and with different frequency behavior arise in biosensors. To improve the performance of sensors and increase the SNR value, it is necessary to study in detail the mechanisms of these noises and identify their effect on the operation of the sensors.

This work is devoted to a detailed study of the types and mechanisms of internal electrical noises, especially low-frequency noise, in specific biosensor architectures. For this purpose, the corresponding electrical equivalent circuits will be constructed and expressions for the rms noise value will be obtained.

Note that BioFETs several structures (ISFET, EIS, etc.) tend to be operated at low frequencies, where $1 / f$-noise is dominated, therefore, our main attention in the next parts of the paper will be focus mainly on low frequency noise.

\section{Definition and Specification of Internal Current Noises}

Main types of semiconductor based BioFET sensors operated using peculiarities of field-effect, especially properties of depletion layer near the interface of semiconductor-insulator. Those are field-effect biosensors: MIS, EIS and ISFET structures.

Electrical noises both in solids and in aqueous solutions have been well studied. Despite this, when considering specific biosensor architectures, it becomes 
necessary to take into account some specific factors that are usually not taken into account in well-known formulas for noise. These factors are associated with miniaturization (size effects), with the generation of complex physical processes at the interfaces and surfaces of specific materials, etc. In this section, for a complete understanding of the problem, in addition to the already known formulas, we will also present expressions obtained and modernized by us for specific designs of sensors operating in different modes and frequency ranges.

Obvious that sensitivity, selectivity and detectivity of electronic devices determined in general by the internal electrical noise's types, its level and frequency behavior, and consequently by the SNR [30]. Voltage (or current) noise spectral density (NSD) $S_{V}$ (or $S_{i}$ ) usually are presented by the rms value of the fluctuating voltage, $\overline{V^{2}}$, or by the fluctuating current $\overline{i^{2}}$ [31]:

$$
\overline{V^{2}}=S_{V} \Delta f, \overline{i^{2}}=S_{i} \Delta f \text {. }
$$

Here $\Delta f$ is the elementary frequency bandwidth. Usually one assumes $\Delta f=1$ $\mathrm{Hz}$.

Equivalent schematic analogy of a noisy resistor presented in Figure 2 for the case of equivalent voltage noise source (Figure 2(a)) and equivalent current noise source (Figure 2(b)).

Below we will shortly characterize the NSD and sources of the main types of noises in metals, semiconductors, dielectrics, bio liquids and electrolyte mediums.

The detailed analysis shows that main types of noises in bio- and chemical sensors can be classified as follows [24] [32] [33] [34] [35] [36].

\subsection{Main Types of Noises in Bio- \& Chemical FET Based Sensors}

1) Noise generated in solid state region:

- Thermal noise;

- Generation-recombination (g-r) noise in the space charge region at the substrate-channel interface;

- $1 / f$ and g-r noises generated due to trapping and detrapping on the semiconductor/insulator interface;

- Hooge's bulk $1 / f$-noise;

- Channel $1 / f$-noise.

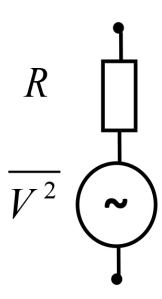

(a)

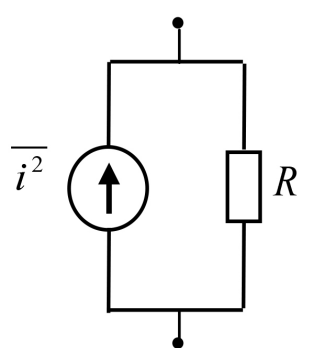

(b)

Figure 2. Equivalent schematic analogy of a noisy resistor $R$. 
2) Electrochemical noise associated with the ion/membrane interactions:

- Thermal noise;

- Shot or Schottky noise;

- $1 / f$-noise in corrosive interfaces;

- Spurious noise.

3) Noise generated in the solution and at the reference electrode as well as noise resulting from the fluctuations of the biasing elements:

- Bulkthermal noise;

- Diffusion layer thermal noise;

- Biological noise.

Thermal noise is the electronic noise generated by the thermal motion of the charge carriers (electrons in conductors, electrons and holes in semiconductors, ions in aqueous solution, bio liquids, electrolytes and dielectrics) inside an electrical conductor at equilibrium, which happens regardless of any applied voltage. Thermal noise is present in all electrical circuits, and in sensitive electronic equipment such as sensors can drown out weak signals, and can be the limiting factor on sensitivity of electrical devices. In an ideal resistor it is approximately white, meaning that the power spectral density is nearly constant throughout the frequency spectrum [31]:

$$
S_{V}^{T}=4 k T R \text { or } S_{i}^{T}=4 k T G,
$$

where $T$ is the thermodynamic temperature, $R$ is the resistance and $G$ is the conductance of the sample.

Those frequency independent noise spectra represent a simplification. An accurate calculation based on a quantum mechanics model gives

$$
S_{V}^{T}(f)=4 R h f\left(\frac{1}{2}+\frac{1}{\mathrm{e}^{h f / k T}-1}\right),
$$

so that Equation (3) is basically only valid for $h f \ll k T$, i.e. for "low" frequencies and high temperatures ( $h$ is the Plank's constant). However, the quantum noise for $h f \gg k T$ has to be considered basically only for frequencies very much higher than in radio frequency and microwave application of concentration of non-equilibrium carriers during the random g-r and trapping-detrapping processes as on the interface impurity states and site bindings, as a result of the bulk random g-r processes.

Generation-recombination noise. NSD of the g-r noise determined by the following expression:

$$
S_{V}^{g-r}=\frac{S_{0}}{1+(\omega \tau)^{2}} .
$$

Here $S_{0}$ is the some constant depending on the semiconductor bulk and surface properties, $\omega=2 \pi f$ is the cyclic frequency, $\tau$ is the time constant (for bulk semiconductors usually fluctuating minority carriers' life time). Generation-recombination noises spectra are described by the Lorentzians. 
Biological noise conditioned by the random fluctuation of the number of captured particles/molecules in acqeous solution. Biological processes, such as protein synthesis or trafficking, undergo random fluctuations, "noise", that are often detrimental to reliable information transfer, but can also constitute opportunities for more efficient cellular computations.

Cellular noise is often investigated in the framework of intrinsic and extrinsic noise. Intrinsic noise refers to variation in identically-regulated quantities within a single cell: for example, the intra-cell variation in expression levels of two identically-controlled genes. Extrinsic noise refers to variation in identically-regulated quantities between different cells: for example, the cell-to-cell variation in expression of a given gene. The main source of stochastic variability on the cellular level is the intrinsic thermal fluctuations of biochemical reactions driving gene expression, signaling, cell cycle, motility, etc. These reactions occur through random collisions and transient binding of various molecular species within a single cell. Cellular noise is random variability in quantities arising in cellular biology. For example, cells which are genetically identical, even within the same tissue, are often observed to have different expression levels of proteins, different sizes and structures. These apparently random differences can have important biological and medical consequences [37] [38] [39]. Cellular noise was originally, and is still often, examined in the context of gene expression levels either the concentration or copy number of the products of genes within and between cells. As gene expression levels are responsible for many fundamental properties in cellular biology, including cells' physical appearance, behaviour in response to stimuli, and ability to process information and control internal processes, the presence of noise in gene expression has profound implications for many processes in cellular biology. For biological noise in [34] predicted a Lorentzian NSD (like g-r noise) with amplitude proportional to the number of particles/molecules in the solution.

Shot noise. Shot noise is a form of noise that arises because of the discrete nature of the charges carried by charge carriers, electrons, holes or ions. When looking at what is shot noise, it can be seen that it is particularly obvious when current levels are low. This is because the statistical nature of the current flow together with the discrete charge levels is more apparent. The continuous flow of these discrete pulses gives rise to almost white noise. There is a cut-off frequency which is governed by the time it takes for the electron or other charge carrier to travel through the conductor. This noise depends on the current flowing and is independent of temperature. The current through the space charge areas of a semiconductor junction (source-channel or drain-channel in BioFET sensors) are composed of many individual current impulses, due to the transport of individual charge carriers. Since this motion of carriers is statistical, we always have, besides the expected dc current $I$, also an ac noise component. With the assumption of individual, rectangular current impulses of the width $\tau$ for every charge component, we can calculate an NSD as following: 


$$
S_{i}^{S-N}=2 e I \times \frac{\sin ^{2}(\pi f \tau)}{(\pi f \tau)^{2}} .
$$

For low frequencies, there is $\sin (x) / x \propto 1$ and we get the commonly used expression for the shot noise

$$
S_{i}^{S-N}=2 e I .
$$

Spurious noise. To discuss digitally-based noise, we need to modify the traditional definition of noise. "Noise is almost always defined as being random, while digitally-based "noise" is deterministic, that is, it can be computed mathematically" [40]. It can be argued that this noise sources are, in fact, all spurious signals, determined by the repetition rate, rise/fall time, amplitude and waveform shape of the electronic signal at various points in the digital circuit. However, the number of these discrete spurious signals is sufficiently large that the net effect is much like random noise, not just a collection of well-defined spurs.

Usually the time constants involved in the detection of biological and chemical species in bio-liquid or electrolyte medium via field effect are relatively large. Therefore, it would be expected that LF (or flicker) noise is more critical than other types of noises in FET based bio- and chemical sensors.

Heerema with coauthors present an extensive study of the $1 / f$-noise in the ionic current through graphene nanopores and compare it to noise levels in silicon nitride pore currents [41]. They find that the $1 / f$-noise magnitude typically two orders of magnitude higher than for silicon nitride pores. This significantly lowers the SNR ratio in DNA translocation experiments. From examining the noise for pores of different diameters and at various salt concentrations, authors find that in contrast to silicon nitride pores, the $1 / f$-noise in graphene pores does not follow Hooge's relation. From studying the dependence on the buffer $\mathrm{pH}$, they also show that the increased noise cannot be explained by charge fluctuations of chemical groups on the pore rim.

Low frequency noise conditioned by the random fluctuations of concentration or mobility of non-equilibrium carriers, ions and charged molecules in aqueous solution, by the trapping-detrapping processes on the free energetic states on the interface surface, as well as by the electron-phonon interactions in the bulk of semiconductor, and by the fluctuation of electron's and phonon's distribution functions. For the BioFET sensors LF NSD can be determined by the Hooge's model, McWhorter or correlated number-mobility fluctuation model and charge fluctuation model.

The main mechanisms of the formation of $1 / f$-noise in semiconductors (active part of FETs) presented in [27] [34] [42]-[55]. The basic $1 / f$-noise theories and models have been proposed to explain the phenomenon in FET based devices: the carrier density or number fluctuation model introduced by McWhorter [56], the carriers mobility fluctuation model proposed by Hooge empirical relation [57], electron-phonon interaction model [51] [53] [54] [58] 
and charge fluctuation model [58] [59] proposed by us.

1) Hooge model [45] [46] [47] [48] [54] [60].

In his paper [45] Hooge proposed that $1 / f$-noise is essentially a bulk phenomenon. Working with metal films he championed an empirical relation for 1/ $f$-noise in terms of resistance fluctuations, where the spectral density of the resistance is $[60]$ :

$$
R_{n}^{2}=\frac{\alpha_{H}}{N} \frac{R^{2}}{f} .
$$

Here $N$ is the total number of free carriers in the bulk, and $\alpha_{H}$ is known as "Hooge's constant" an empirical parameter. This equation fits his data for metal films very well. In [61] Vandamme developed Hooge's theory for MOSFET devices, starting from the lattice scattering theory presented in [62], calculating

$$
I_{n}^{2}=\frac{\mu_{0}}{\mu_{e f f}}\left(\frac{\mu_{0}}{\mu_{l}}\right)^{2} \frac{q \alpha_{H} I_{D}^{2}}{w l\left(V_{G S}-V_{t h}\right)} \frac{1}{f},
$$

where $\mu_{0}$ is the low-field mobility of the carriers, $\mu_{l}$ is the mobility if only lattice scattering exists,

$$
\mu_{e f f}=\frac{\mu_{0}}{1+\theta\left(V_{G S}-V_{t h}\right)},
$$

$\theta$ is the mobility attenuation factor, $V_{G S}$ and $V_{\text {th }}$ are gate-source and threshold voltages. Again, significant bias dependences can be explained by changes in the assorted mobility parameters.

For modeling $S_{i}(f)$ dependencies barely will be useful Hooge's universal formula of current noises for materials and structures with macroscopically homogeneous current density [45]:

$$
S_{i}(f)=\frac{\alpha_{H} I^{2}}{N_{t o t} f^{\gamma}},
$$

where Hooge's parameter $\alpha_{H}$ for semiconductors typically $\alpha_{H} \approx 2 \times 10^{-3} \quad$ [42] [44] [46] [47] [48] [54], $N_{\text {tot }}$ is the total number of electrons in the sample (means the total number of moving carriers in the sample), $I$ is the current, magnitude of $\gamma$ close to 1 . Unfortunately, Hooge's formula does not give opportunity to take into account the processes take place in the bio-liquid or electrolyte medium of the BioFET sensors on the base of bare EIS and ISFET structures [63] [64].

\section{2) McWhorter's or Correlated Number-Mobility Fluctuation Model}

McWhorter, working with germanium filaments at MIT Lincoln Laboratory in 1957 proposed that flicker noise is primarily a surface effect [56]. He cites a number of experiments that showed that the $1 / f$-noise in germanium is dependent on the ambient atmosphere of the filament. He writes, "recent results now leave little doubt that the noise is predominantly, if not entirely, a surface phenomenon." Thus, the McWhorter number fluctuation ( $\Delta n$ ) theory states that flicker noise is generated by fluctuations in the number of carriers due to 
charge trapping in surface states. McWhorter obtained the necessary $1 / f$ spectrum by assuming that the time constant $\tau$ of the surface states varied with a $1 / \tau$ distribution. Christensson et al. [65] [66] were the first to apply the McWhorter theory to MOSFETs, using the assumption that the necessary time constants are caused by the tunneling of carriers from the channel into traps located inside the oxide.

In the correlated number-mobility fluctuation theory NSD of the flat-band voltage fluctuation can be described as [27]

$$
S_{V, F B}(f)=\frac{e^{2} k T N_{t}}{w l \beta C^{2}} \frac{1}{f} .
$$

Here, $w$ and $l$ are width and length of the insulator gate, $\beta=\frac{2}{\hbar} \sqrt{2 m^{*} \Phi_{B}}$ is the McWhorter's or tunneling parameter ( $m^{*}$ is the effective mass of electrons, $\Phi_{B}$ is the tunneling barrier height seen by electron at the interface), $N_{t}$ is the oxide equivalent trap density in $\mathrm{eV}^{-1} \mathrm{~cm}^{-3}, C$ is the cumulative capacitance.

The McWhorter's model attributes the origin of $1 / f$-noise to the random fluctuation on the number of carriers in the channel due to fluctuations in the surface potential ${ }^{1}$. The fluctuations are caused by rapping and detrapping of carriers by energetic traps located near the $\mathrm{Si}_{-} \mathrm{SiO}_{2}$ interface [67] [68]. In the saturation regimes the drain current NSD is calculated as:

$$
S_{\text {id }}(f)=\frac{K_{F} I_{d s}}{C_{o x} L^{2}} \frac{1}{f} .
$$

where $K_{F}$ is a quality independent on bias but dependent on sensor fabrication process.

The Hooge's empirical model attributed $1 / f$-noise to carriers' mobility fluctuations, due to carrier interactions with crystal lattice fluctuations [45] [57]. At saturation

$$
S_{i d}(f)=\frac{\alpha_{H} q \mu_{f} I_{d s}\left(V_{g s}-V_{t h}\right)}{L^{2}} \frac{1}{f},
$$

where $\alpha_{H}$ is Hooge $1 / f$-noise parameter (Hooge's parameter), $\mu_{f}$ is carriers' effective mobility.

For low noise applications the level of thermal and $1 / f$-noises must be sufficiently low.

Based on the number fluctuation noise model [44] the calculations of the $1 / f$ -noise spectra for ISFET has been presented in [69]. At saturation

$$
S_{i d(f)}=\frac{q^{2} \mu N_{o t} I_{d s a t}}{C_{o x} L^{2}} \frac{1}{f},
$$

and at sub-threshold

$$
S_{i d(f)}=\frac{q^{4} N_{o t} I_{d s}^{2} C_{i n v}^{2}}{\left(C_{o x}+C_{D}\right)^{4}(k T)^{2} W L} \frac{1}{f}
$$

${ }^{1}$ In further we will show that for DNA sensors the fluctuations in the surface potential is depend on DNA molecules concentration. 
Here $C_{i n v}, C_{o x}$ and $C_{D}$ are the inversion, oxide and depletion layers capacitances per unit area, $N_{o t}$ is the effective oxide traps density per unit area.

Problems of minimization of ISFET noises and results of LF noise measurements are detailed discussed in [70].

\section{3) Charge Fluctuation Model}

We can consider $1 / f$-noise source, $\overline{V^{2}}$, via fluctuation of the oxide traps (free bonds of proton donors $\mathrm{O}^{-}$and proton acceptors $\mathrm{OH}_{2}^{+}$, Figure 2 in [13]) charge density. The charge density of occupied traps $Q_{t}$ can be described as:

$$
Q_{t}=\frac{e N_{o t}}{w l},
$$

where $N_{o t}$ is the number of occupied traps. Then the NSD of the charge fluctuation of occupied traps is:

$$
S_{Q}(f)=\left(\frac{e}{w l}\right)^{2} S_{N_{o t}}(f) .
$$

Spectral density of the number fluctuations of occupied traps can be determined as [44]:

$$
S_{N_{o t}}(f)=\frac{w l N}{f} .
$$

The voltage-fluctuation noise spectral density can be calculated using the expression:

$$
S_{V}(f)=\frac{S_{Q}(f)}{C_{e f}^{2}}=\frac{e^{2} N}{w l C_{e f}^{2}} \frac{1}{f} .
$$

In expressions (10) and (11) $N$ is the equivalent density of traps per unit area at the $\mathrm{SiO}_{2}$ /electrolyte interface, $C_{e f}=\frac{C_{i} C_{d}}{C_{i}+C_{d}}, C_{i}$ is the capacitance of the insulator (oxide) layer and $C_{d}$ is the capacitance of the semiconductor depletion layer.

\subsection{Signal-to-Noise Ratio (SNR)}

SNR is a measure for comparison of the level of a desired signal to the level of background noise. SNR is defined as the ratio of signal power $P_{S}$ to the noise power $P_{N}$. For linear devices SNR can be calculated from expressions:

$$
\begin{aligned}
\text { SNR } & =\frac{P_{S}}{P_{N}}=\frac{I_{S}}{I_{N}}=\frac{I_{S}}{\sqrt{S_{i} \Delta f}} \\
& =\frac{V_{S}}{V_{N}}=\frac{I_{S} R_{\text {tot }}}{\sqrt{S_{V} \Delta f}} .
\end{aligned}
$$

Here $I_{S}\left(V_{S}\right)$ and $I_{N}\left(V_{N}\right)$ are useful signal current (voltage) and noise equivalent current (voltage), correspondingly, $R_{\text {tot }}$ is the total resistance of the sample.

In review [70] different FET structures and detection principles are discussed, 
including label-free and indirect detection mechanisms. The fundamental detection principle governing every potentiometric sensor is introduced, and different state-of-the-art FET sensor structures are reviewed. This is followed by an analysis of electrolyte interfaces and their influence on sensor operation. The successful detection of biomolecules by a BioFET is dictated by the sensor's intrinsic SNR. The detection limit of a traditional BioFET is fundamentally limited by biomolecule diffusion, charge screening, linear charge to surface-potential transduction, and flicker noise ( $1 / f$-noise). In [71] authors demonstrate that $\mathrm{t}$ transistor technology called Negative Capacitor Field Effect Transistor (NCFET) offers nonlinear charge transduction and suppression of flicker noise to dramatically improve the SNR over classical Boltzmann sensors. The SNR improvement by interpreting the experimental results associated with the signal and noise characteristics of $2 \mathrm{D} \mathrm{MoS}_{2}$-based transistors quantify. The combined sensitivity enhancement and noise rejection guarantee a high SNR of the NCBioFET, making this device a promising candidate for realizing advanced integrated nano-biosensors. Most importantly, NCFETs can improve the SNR compared to traditional MOSFETs by reducing the LF flicker noise related to carrier number fluctuations. The results of the analysis show that despite the fundamental limits of charged-based BioFETs, the NCBioFET can improve the limits of label-free detection of biomolecules.

McAndrew with coauthors shows how correlated noise can be implemented in Verilog-A, and presents a new and simple technique to simulate the noise correlation coefficient using only the standard Space noise analyses [72]. An analytic proof is given that the noise contributed by the distributed gate resistance of the MOSFET can be modeled by including a gate resistance of value $R_{g} / 3$ in series with the gate capacitance. Analyses of series and parallel combinations of devices are done to derive fundamental geometric scaling relations for noise. It is shown that gate noise must be distributed between gate-source and gate-drain components by maintaining proper symmetry. In saturation the correlation coefficient between gate and drain noise currents is imaginary $(j \sqrt{5 / 32})$ [72]. For resistors (including both thermal and $1 / f$-noise components) [73]

$$
S_{i}(f)=4 k T G+K_{F} \frac{I^{2}}{W L} \frac{1}{f} .
$$

Numerical and analytical theory of signal and noise of double-gated $\mathrm{pH}$-sensors was provided in [74]. Relatively high noise floor of ultra-scaled NW sensors limits the SNR and the corresponding $\mathrm{pH}$ resolution $\left(\Delta \mathrm{pH}_{\min }\right)$ of such devices. It is defined $\Delta \mathrm{pH}_{\min }$ as being the minimum change in $\mathrm{pH}$ above the noise floor that can be continuously (without signal averaging) detected by the FET-sensor. In this article, authors offer a comprehensive theoretical analysis of double-gated pH sensors, with emphasis on the "so-called" amplified Nernstian response and the SNR. Authors combine the classical theory of the MOSFET [75] and the site-binding model [29] [76] to calculate the signal amplification and the noise levels for DGFETs (Double Gate FET) in various biasing configu- 
rations (i.e., accumulation, depletion, and inversion). For the noise calculation in DGFET sensors, authors account for the noise sources from the top and bottom sides of Si body by assuming that the DGFET can be viewed as two independent MOSFETs with different oxide thicknesses and gate voltages.

In [77] the electrical characteristics and $\mathrm{pH}$ responses of a Si-nanonet ISFET with ultra-thin parylene- $\mathrm{H}$ as a gate sensing membrane was reported. From the LF noise characterization, the SNR was extracted as high as $~ 3400 \mathrm{~A} / \mathrm{A}$ with the lowest noise equivalent $\mathrm{pH}$ value of $\sim 0.002 \mathrm{pH}$. These excellent intrinsic electric$\mathrm{al}$ and $\mathrm{pH}$ sensing performances suggest that parylene- $\mathrm{H}$ can be promising as a sensing membrane in an ISFET-based biosensor platform. The $\mathrm{pH}$ sensitivity of the $\mathrm{pH}$ ISFETs in various buffer solutions and conducted LF noise analysis for potential development in bioassay applications is evaluated. The $\mathrm{pH}$ responses of the devices were evaluated in various $\mathrm{pH}$ buffer solutions. A high $\mathrm{pH}$ sensitivity of $48.1 \pm 0.5 \mathrm{mV} / \mathrm{pH}$ with a device-to-device variation of $\sim 6.1 \%$ was achieved. The LF noise measurements of $\mathrm{pH}$ ISFET were performed in $\mathrm{pH}=7$ buffer solution under various gate bias conditions. The noise spectrum $S_{I D}$ shows a typical $1 / f^{\alpha}$ behavior with the exponential slope $\alpha \propto 1$ in a 3-dec frequency bandwidth of $f_{1}-f_{2}=1-1000 \mathrm{~Hz}$. The SNR was extracted based on the following equation [78]:

$$
\mathrm{SNR}=\Delta I / \sqrt{\int_{f_{1}}^{f_{2}} S_{I D}(f) \mathrm{d} f},
$$

where $\Delta I$ is the drain current change in the range of $\mathrm{pH}=4-10$. In range $1-$ $1000 \mathrm{~Hz} S_{I D}(f) \propto\left(10^{-20} \div 10^{-25}\right) \quad \mathrm{A}^{2} / \mathrm{Hz}\left(V_{g} \propto 0.8 \div 1.2 \mathrm{~V}\right), \mathrm{SNR}_{\text {max }} \propto 3000$ $\mathrm{A} / \mathrm{A}$ at $V_{g} \propto 1.0 \mathrm{~V}$.

Our research in the field of mechanisms of LF noise in semiconductors, semiconductor devices and BioFET sensors is devoted to a number of works (e.g. [50] [53] [54] [58] [59] [62] [79] [80] [81] [82]).

The main sources of electron mobility fluctuations in semiconductors were analyzed in [54], the theory of $1 / f$-fluctuations of the lattice mobility of current carriers in homogeneous semiconductors, the problems of space confined and bulk temporal fluctuations of acoustic phonons and relationship between models of $1 / f$-noise in semiconductors are developed, analyzed and presented in [58]. Phonons interface percolation dynamics detailed investigated in [51]. Influence of long-wave acoustic longitudinal-phonon percolation dynamics on $1 / f$-type noise level is modeled for homogeneous, non-degenerated and bounded semiconductors. Phonons percolation from semiconductor media to environment regions via so called "refraction points" of phonons' wave vector phase space is modeled within framework of the bulk mechanism of electron lattice mobility fluctuation. On the base of this mechanism it is shown, that semiconductor surface is the source of suppression of $1 / f$-noise [83]. Phonon mechanism of equilibrium fluctuation of carrier's mobility and properties of $1 / f$-noise analyzed also in [53].

Brief overview of the basic tendencies of development of nanoscale (bio-)chemical 
sensors is presented in [79]. Last years' experimental data and theoretical models for LF excess noises are summarized and analyzed; in particularly works in the field of sensors functionalized by the nanoparticles, nanotubes (NT) and nanowires (NW) are discussed. Nanoscale FETs electrolyte gate field-effect devices and capacitive EIS structures were discussed. Main mechanisms of excess noises formation, behavior and peculiarities of the NSD, and the Hooge parameter were analyzed. Role of the functionalization (with several nanoparticles, NTs and NWs) on the essential reduction of $1 / f$-noise level is discussed. On the base of the analyses of electrical, physical, bio-chemical and other characteristics of the nanoscale sensors based on the NTs and NWs do some conclusions [79]:

- Low-frequency noise spectral density generally expressed as

$$
S_{v}=A \frac{V^{2+\beta}}{f^{\gamma}} .
$$

- The noise amplitude (parameter $A$ ) reflects the sample quality and increases with decreasing device size and depends on many parameters of material, its structure, sizes, NTs bulk and surface physical and chemical conditions, from its fabrication method.

$$
A \propto R, \quad A \propto \frac{1}{N}, \quad A \propto \frac{1}{L} .
$$

where $R$ is the device resistance, $N$ is the number of atoms or carriers in the system, $L$ is the sample length $(N \propto L)$. $A=1 \times 10^{-11} R$. Parameter $A$ varies within $10^{-13}$ up to $4 \times 10^{-4}$.

- The size scaling is incorporated in Hooge's empirical law $A=\frac{\alpha_{H}}{N}$.

- In the linear regime $1 / A \propto\left|V_{g}-V_{t h}\right|$ if noise is due to mobility fluctuations and $1 / A \propto\left|V_{g}-V_{t h}\right|^{2}$ if noise is due to number fluctuations.

- Parameter $\beta=0$ is expected for pure resistance fluctuation in ohmic conductors. The $\gamma \neq 1$ behavior is associated with nonlinear characteristics.

- Excess noise with a slope different from unity $(\gamma \neq 1)$ can be explained by a superposition of a few Lorentzians.

In [58] charge fluctuation LF noise model is modifying for an EIS structure. Physical processes take part in the semiconductor, insulator and electrolyte medium responsible for LF charge fluctuation are discussed. The complete electrical noise equivalent scheme for EIS structure is designed. Dependencies of NSD from charge fluctuation connected with processes on the electrolyte-insulator, insulator-semiconductor interfaces and bulk semiconductor are determined. It is shown that in the bare EIS structure a number of binding sites of the insulator surface actively take part in the trapping-detrapping processes with the ions and molecules situated in the electrolyte. Usually these are very slow processes. The capacitance $C_{o x}$ and captured charge will be slowly fluctuated. In the other hand capacitance of the semiconductor depletion region also depend on charge fluctuation of insulator-semiconductor and insulator-electrolyte interfaces and fluctuated with them via surface potential. Generally, noise determined by the 
modulation of semiconductor depletion region capacitance and surface potential due to charge fluctuation at the insulator-electrolyte interface. Low-frequency noise in an EIS structure functionalized with a multilayer of polyamidoamine (PAMAM) dendrimer and single-walled carbon nanotubes (SWNT) is studied in [64]. The NSD exhibits $1 / f^{\gamma}$ dependence with the power factor of $\gamma \approx 0.8$ and $\gamma=0.8 \div 1.8$ for the bare and functionalized EIS sensor, respectively. The gate-voltage NSD was practically independent on $\mathrm{pH}$ value of the solution, and is increased with increasing the gate voltage or gate-leakage current. It has been revealed that functionalization of an EIS structure with the PAMAM/SWNTs multilayer leads to an essential reduction of $1 / f$-noise. To interpret the noise behavior in bare and functionalized EIS devices, a gate-current noise model for capacitive EIS structures based on an equivalent flat band-voltage fluctuations concept has been developed [64]. The experimentally observed gate-voltage dependence of the noise explained by the gate-voltage-dependent changes in the occupancy of the oxide trap levels resulting in a modulation of the conductivity of current paths or charge carriers passing through the EIS structure.

LF noise spectroscopy at nanoscale and noise reduction in BioFET sensors functionalized with carbon NT multilayers areee detailed analyzed in [80] [82]. Transport properties of Si NW double gated BioFET sensors were investigated involving noise spectroscopy and current-voltage characterization [81]. Transport and noise properties of NW FET structures were investigated as in sensor configuration in an aqueous solution with different $\mathrm{pH}$ values. Furthermore, it is studied channel length effects on the noise, and $\mathrm{pH}$ sensitivity (Figure 3 ). The magnitude of the $\mathrm{pH}$ sensitivity increases with the increase of channel length approaching the Nernst limit value of $59.5 \mathrm{mV} / \mathrm{pH}$. It is demonstrated that dominant $1 / f$-noise can be screened by the generation-recombination plateau at certain $\mathrm{pH}$ of the solution. It is shown that the measured value of the slope of $1 / f$-dependence on the current channel length is 2.7 which is close to the theoretically predicted value of 3 .

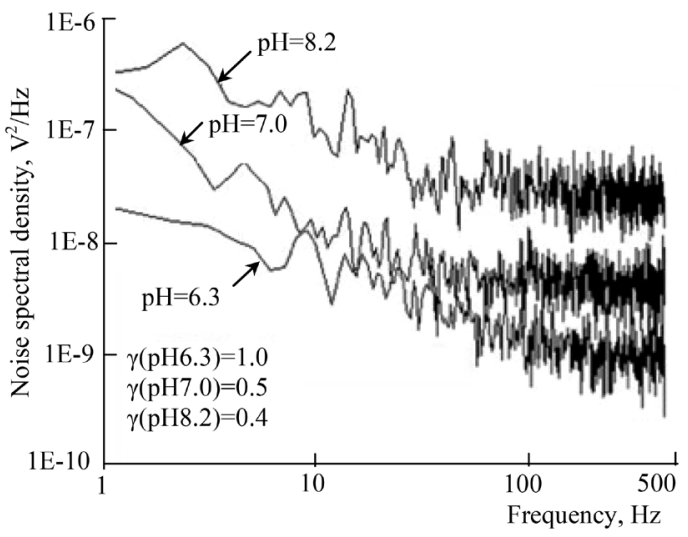

Figure 3. Noise spectra of NW double gated FET sensor, measured in different $\mathrm{pH}$ solutions Spectral dependence of LF noise for NW with length $I=10 \mu \mathrm{m}$ measured at $300 \mathrm{~K}$ and several $\mathrm{pH}$ values: $6.3,7.0$, and 8.2 at the back gate voltage $-5 \mathrm{~V}$ and front gate voltage $-1 \mathrm{~V}$. Adopted from [81]. 


\section{Noises in BioFET Structures (MIS, EIS, ISFET)}

In this section, for the first time, complete equivalent electrical circuits of some biosensors are constructed, taking into account the characteristics of a semiconductor, dielectric, aqueous solution, analyte and reference electrode. On the basis of these schemes, the spectral densities of the internal electrical noises (or rms values) were calculated. In these calculations, we used both well-known formulas (for example, for thermal noise) and formulas that we have modernized and obtained for specific investigated cases and operating modes. We also used the results of studies by other authors. These expressions and formulas can be found both in ours and in articles by other authors listed in the cited references.

For analyzing the noise properties, we need model equivalent electrical schemes of the elements of investigated structures. Below we will use the standard equivalent schematic analogy of a noisy resistor $R$ consisting serial connected voltage generator via average square of the fluctuating voltage $\overline{V^{2}}$, or by the parallel connected current generator via average square of the fluctuating current $\overline{i^{2}}$ (Figure 2(a), Figure 2(b)).

\subsection{MIS Structures}

On Figure 4 the schematic picture of MIS structure is presented. Electrical and other characteristics of such type structures detailed are investigated [42] [48] [84] [85].

Electrical equivalent scheme for noises analyses for this case can be presented as the serial connection of semiconductor and insulator layers (see Figure 5). Each part has its bulk resistance ( $R_{S}$ and $R_{i}$, correspondingly for semiconductor and insulator layers) and parallel connected capacitances $\left(C_{d}\right.$ and $C_{i}$, correspondingly for semiconductor depletion layer and insulator), $\overline{V_{S}^{2}}$ and $\overline{V_{i}^{2}}$ are the noise sources for semiconductor and insulator, correspondingly, $\frac{V_{F B}^{2}}{V_{F}^{2}}$ is the noise source conditioned by the flat-band voltage fluctuation. Total noise can be presented as the sum

$$
\overline{V_{M I S}^{2}}=\overline{V_{i}^{2}}+\overline{V_{S}^{2}}+\overline{V_{F B}^{2}},
$$

where

$$
\overline{V_{S}^{2}}=4 k T R_{S b}+\frac{4 k T R_{S d}}{1+\left(\omega R_{S d} C_{d}\right)^{2}}, \overline{V_{i}^{2}}=4 k T R_{i}, \overline{V_{F B}^{2}}=\frac{e^{2} k T N}{\gamma w l C_{e f}^{2}} \frac{1}{f}, R_{S}=R_{S b}+R_{S d},
$$

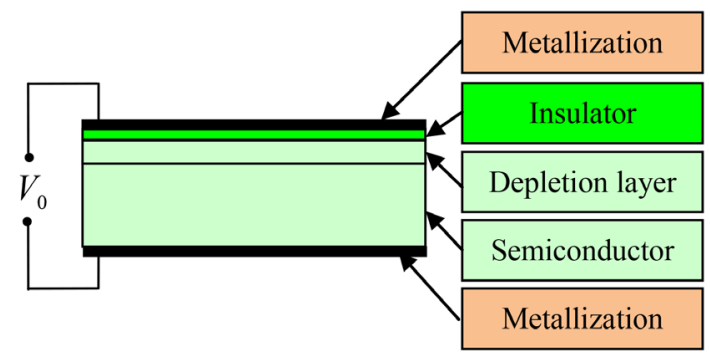

Figure 4. MIS structure (Al-pSi-SiO $2-\mathrm{Al})$. 


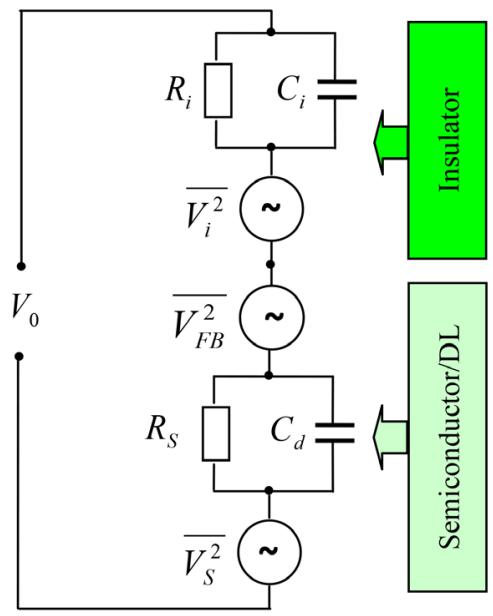

Figure 5. Electrical scheme for MIS noise analyses.

$R_{S b}$ and $R_{S d}$ are resistances of the bulk region and depletion layer of the semiconductor, correspondingly. Note that second term in right hand of $\overline{V_{S}^{2}}$ is the g-r part of the noises, conditioned by the g-r processes in the depletion region. The noise source $\overline{V_{F B}^{2}}$ characterizes fluctuation processes which take part on the interface of the depletion region and insulator. Thus

$$
\begin{aligned}
\overline{V_{M I S}^{2}} & =4 k T\left(R_{S b}+R_{i}\right)+\frac{e^{2} k T N_{t}}{\gamma w l C_{e f}^{2}} \frac{1}{f}+\frac{4 k T R_{S b}}{1+\left(\omega R_{S d} C_{d}\right)^{2}} \\
& \approx 4 k T R_{i}+\frac{e^{2} k T N_{t}}{\gamma w l C_{e f}^{2}} \frac{1}{f}+\frac{4 k T R_{S b}}{1+\left(\omega R_{S d} C_{d}\right)^{2}},
\end{aligned}
$$

as $R_{i} \gg R_{S}$. Therefore, noises consist of the thermal, flicker and g-r components.

\subsection{EIS Structures}

Schematic picture of EIS structure and its electric equivalent scheme are presented in Figure 6 and Figure 7. On the base of detailed analyses of several properties of EIS structures (see, e.g. [24] [27] [33] [36] [55] [86]), the electrical scheme for noises analyses for the case presence of DNA molecules (as a biomolecule) can be presented as on Figure 9. Main noise sources are conditioned by the flat-band voltage fluctuation, semiconductor bulk resistance fluctuation and electrolyte bulk resistance fluctuation. Comparable with the case of MIS structure, the following additional new sources of noises are presented on Figure 7:

$$
\overline{V_{c h}^{2}}=\frac{e^{2} N}{A C_{e f}^{2}} \frac{1}{f} ; \overline{V_{D}^{2}}=4 k T R_{D} ; \overline{V_{R E}^{2}}=4 k T R_{R E} ; \overline{V_{b}^{2}}=4 k T R_{b} ; \overline{V_{D N A}^{2}}=\frac{K_{D} V_{D}^{\alpha}}{f^{b}} .
$$

Here $\overline{V_{c h}^{2}}$ is the spectral density of the charge fluctuation on the insulator-electrolyte interface [43], $\overline{V_{D}^{2}}$ is the spectral density of the thermal noise of charge transfer resistance fluctuations; $R_{D}$ is the charge transfer resistance across electrolyte; $\overline{V_{R E}^{2}}$ is the spectral density of the thermal noise of the reference electrode resistance fluctuations; $R_{R E}$ is the resistance of reference elec- 
trode. $\overline{V_{b}^{2}}$ is the bulk electrolyte resistance $\left(R_{b}\right)$ noise. The bulk electrolyte resistance can be approximated by the spreading resistance $R_{b} \approx \frac{1}{K} \sqrt{\frac{\pi}{w l}}, K$ is the electrolyte conductivity [24]. The noise source $\overline{V_{D N A}^{2}}$ represents an anticipated noise source, $V_{D}$ is the potential on the DNA layer, $K_{D}$ some parameter, $\alpha$ and $b$ is the some exponents. This is due to the random motion of the immobilized DNA probes within the electrolyte. This motion can couple to the semiconductor channel and cause random fluctuations in the carriers. It is expected that this noise source would depend on the potential across the DNA layer, since a higher potential could potentially further immobilize the probes and cause less noise [24].

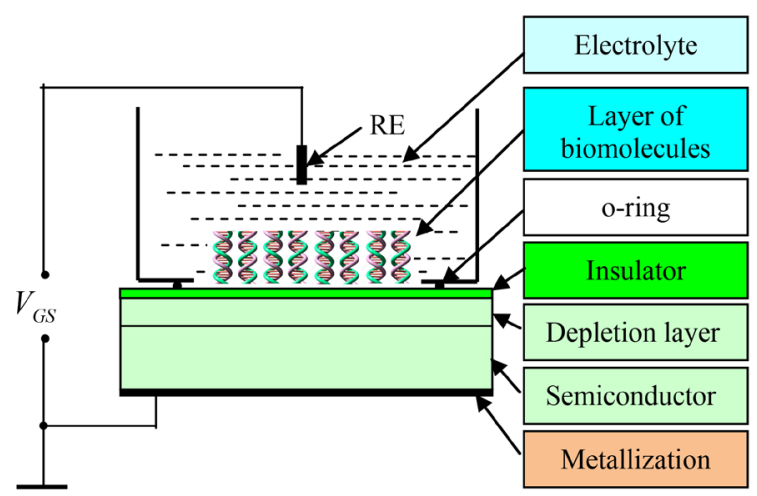

Figure 6. Schematic picture of EIS structure.

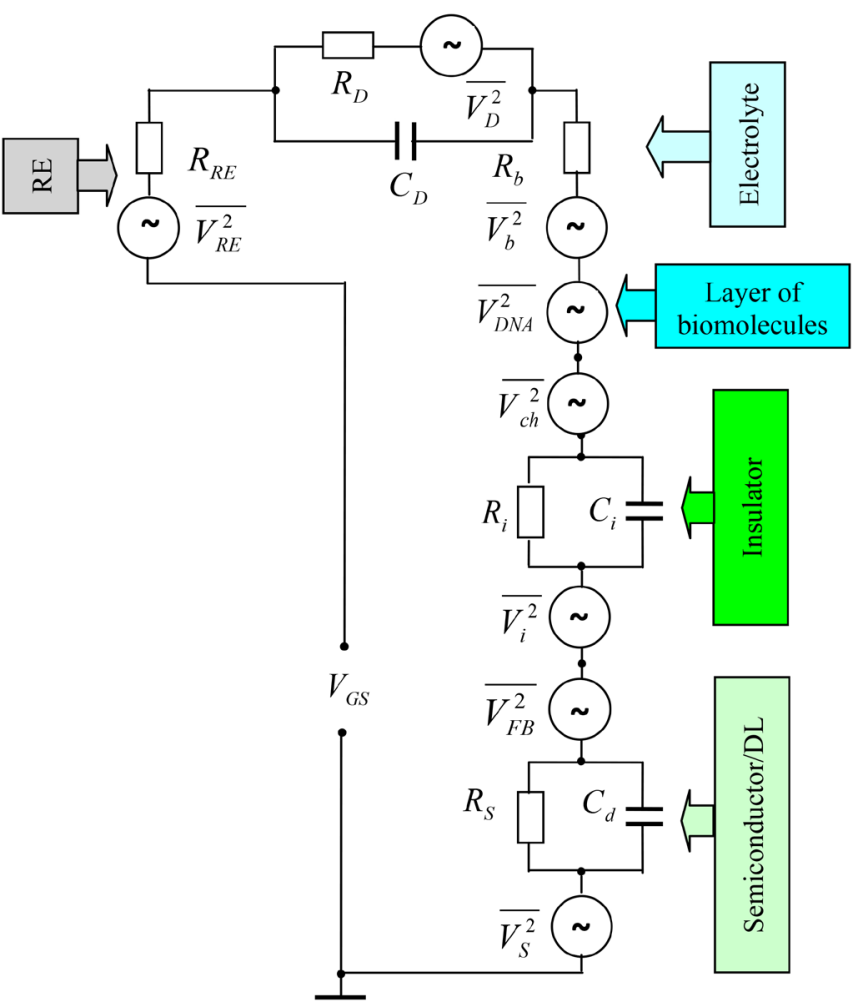

Figure 7. Electrical scheme for EIS noise analyses. 
Therefore, for EIS structure we can write

$$
\begin{aligned}
\overline{V_{E I S}^{2}}= & 4 k T\left(R_{S b}+R_{i}+R_{b}+R_{D}+R_{R E}\right)+\frac{K_{D} V_{D}^{\alpha}}{f^{b}} \\
& +\frac{e^{2} N}{w l C_{e f}^{2}}\left(1+\frac{k T N_{t}}{\gamma N}\right) \frac{1}{f}+\frac{4 k T R_{S b}}{1+\left(\omega R_{S d} C_{d}\right)^{2}} .
\end{aligned}
$$

Taking into account that

$$
R_{i} \gg R_{S}+R_{b}+R_{D}+R_{R E},
$$

for the NSD of the EIS biosensor we get

$$
\overline{V_{E I S}^{2}} \approx 4 k T R_{i}+\frac{K_{D} V_{D}^{\alpha}}{f^{b}}+\frac{e^{2} N}{w l C_{e f}^{2}}\left(1+\frac{k T N_{t}}{\beta N}\right) \frac{1}{f}+\frac{4 k T R_{S b}}{1+\left(\omega R_{S d} C_{d}\right)^{2}} .
$$

\subsection{ISFET/MOSFET Structures}

For the sensors on the base of such structures we have important difference. Schematic picture and electrical scheme of the ISFET/MOSFET structure presented on Figure 8 and Figure 9, correspondingly. There we have two "current channels" [24] [33] [43] [44] [78] [79] [87] [88]: 1) vertical leakage current or equivalent voltage noise scheme (Metal-Semiconductor-Insulator-Electrolyte-RE, e.g. EIS, which electrical equivalent scheme presented on Figure 7), and 2) horizontal equivalent current noise scheme Source-Channel-Drain, (Figure 9, below).

Source-drain current noise can be successfully described by the Hooge's $1 / f$ noise, flat-band voltage fluctuation, semiconductor potential fluctuation and depletion region resistance fluctuation.

The NSD of the drain current noise in MOSFETs using also results of [24] can be presented as:

$$
\begin{aligned}
\overline{i_{D S}^{2}}= & 4 k T \mu_{e f} C_{e f} \frac{w}{l}\left(V_{F B}^{\prime}+\varphi_{S 0}-V_{A}-\varphi_{00}-\frac{\sqrt{2 e \varepsilon_{S} N_{D}}}{C_{i}}\right) \\
& +\frac{e^{2} k T N_{t}}{\gamma w l C_{e f}^{2}}\left(g_{m}+\alpha \mu_{e f} C_{e f} I_{D}\right)^{2} \frac{1}{f}+\frac{\omega \tau I_{0}^{2}}{1+(\omega \tau)^{2}} .
\end{aligned}
$$

Here first term describes thermal, second term, flicker and third term, g-r part of the noise.

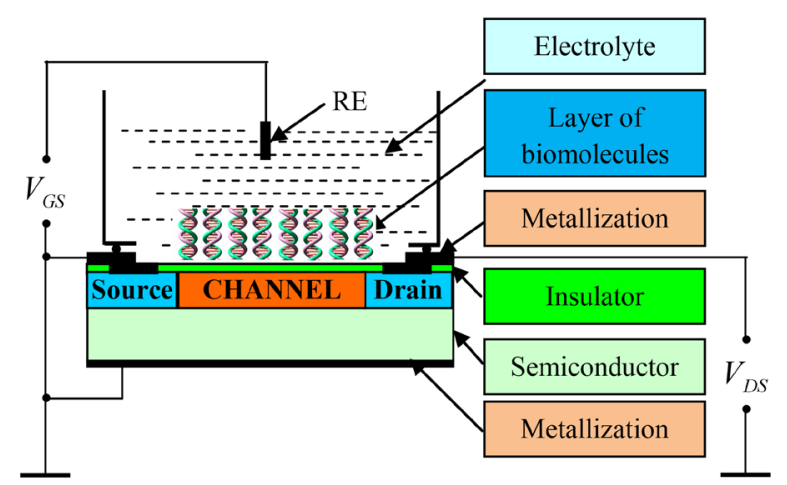

Figure 8. MOSFET/ISFET schematic picture. 


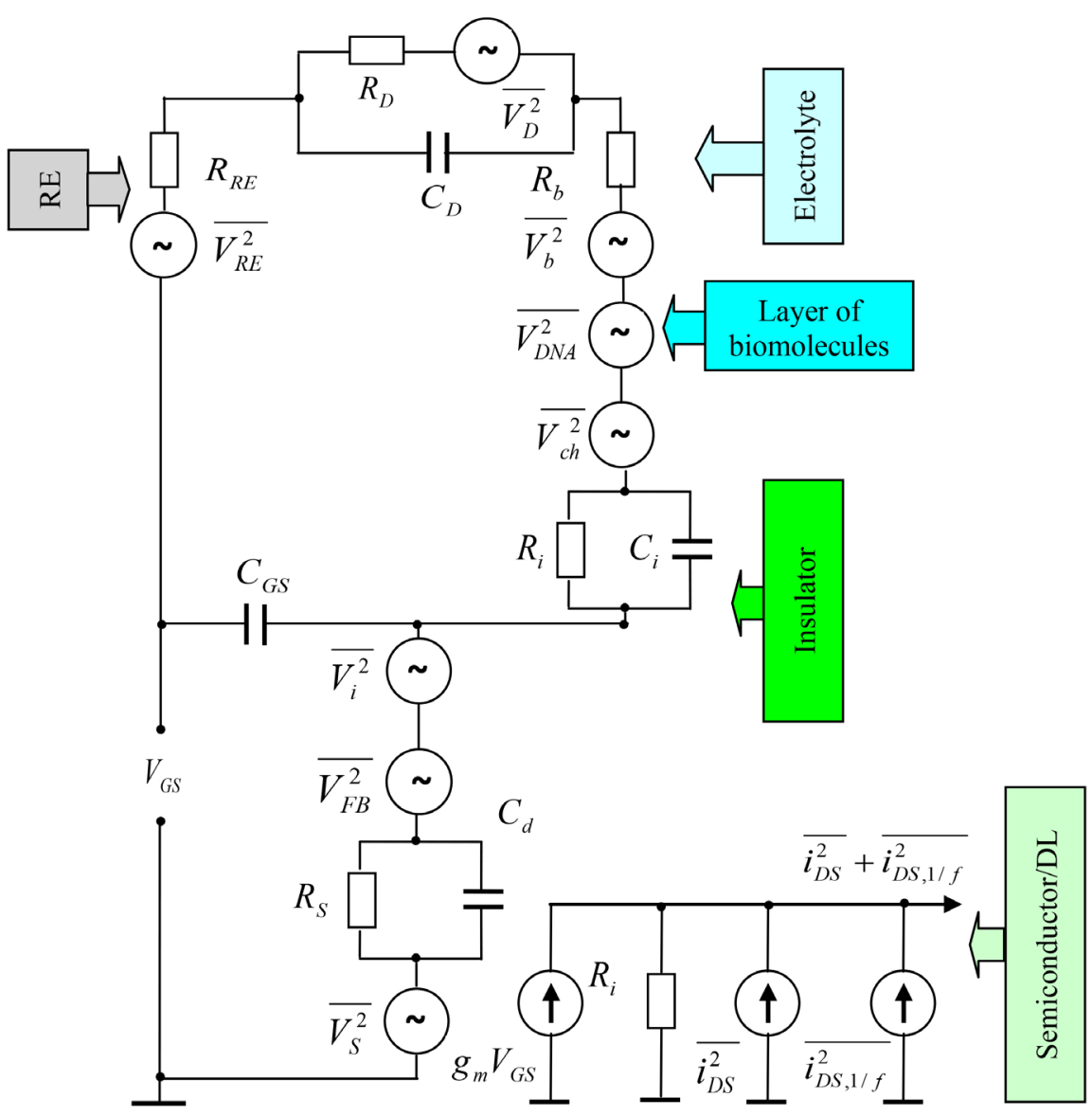

Figure 9. Electrical scheme for ISFET/MOSFET noise analyses (non faradaic electrode).

The NSD of the drain current noise in a load resistor $R_{L}$ connected between source and drain in the linear regime approximated as [79] [88]:

$$
\overline{i_{D S}^{2}}=\frac{\overline{i_{C h}^{2}}+g_{C h}^{2} R_{D S}^{2} \overline{i_{R_{d S}}^{2}}}{\left[1+g_{C h}\left(R_{d S}+R_{L}\right)\right]^{2}} .
$$

According to Hooge's empirical mobility fluctuation model for elementary bandwidth $\Delta f=1 \mathrm{~Hz}$

$$
S_{i}(f)=\overline{i_{D S, 1 / f}^{2}}=\frac{e \alpha_{H} i_{D S}}{w l C_{o x}\left(V_{G S}-V_{T}\right)} \frac{1}{f}=\frac{e \alpha_{H} \mu_{e f} i_{D S} V_{D S}}{l^{2}} \frac{1}{f} .
$$

In (12)-(13) $\alpha$ is Coulomb scattering coefficient; $C_{d}$ is the capacitance of the diffusion layer; $C_{e f}$ is the effective capacitance per unit area, consisting of the insulator and any functionalization layer; $C_{d l}$ is the depletion layer capacitance; $I_{0}$ in (13) is the any parameter; $R_{L}$ is the load resistor resistance; $R_{S d}$ is the resistance of semiconductor bulk and depletion layer; $R_{d S}$ is the series resistances; $\overline{i_{C h}^{2}}$ is the noise contribution from the channel with conductance $g_{C h}$, $\overline{V_{S}^{2}}$ is the semiconductor bulk resistance voltage noise; $R_{c t}=\frac{k T}{Z e}\left|\frac{1}{i_{T}}\right|$ is the charge transfer resistance [34]; $Z$ is the atomic number, $i_{T}$ is the total redox 
current; $g_{m}$ is the transconductance; $\overline{V_{W 1}^{2}}=4 k T\left(R_{c t}+R_{W 1}\right) ; \overline{V_{W 2}^{2}}=4 k T R_{W 2}$; $R_{W 1}$ and $R_{W 2}$ are the Warburg impedances [24]; $\bar{i}_{1}^{2}=2 Z e i_{T} K(\omega)$ is the non-equilibrium noises source describe specific mass transfer effect at the electrolyte-electrode interface; $K(\omega)$ is the corresponding coefficient [33].

As we can see noise spectral density for all investigated cases consist three obligatory components: thermal, low-frequency and generation-recombination. Depending on the special experiment conditions either type of noises will be dominated in the appointed frequency interval. For example in EIS functionalized with PAMAM SWNT $1 / f$-noise dominates over thermal noise lower 10 $\mathrm{Hz}$ [63] [64], drain current NSD for the silicon-on-insulator (SOI) p-MOSFET biased back gate voltages $1 / f$-noise dominates in the region up to $500 \mathrm{~Hz}$ [79], for ISFETs at the buffer solutions $\mathrm{pH} 7$ and $\mathrm{pH} 10$ above a corner frequency $\sim 1$ $\mathrm{Hz}$ up to $400 \mathrm{~Hz}$ the measured spectra correspond to $1 / f$-noise and below this corner frequency the measured spectra correspond to $1 / f^{2}$, in $\mathrm{n}$ - and p-channel dynamic-threshold MOSFETs on SOI substrate NSD has both quasi $1 / f$ and Lorentzian spectra up to $10^{4} \mathrm{~Hz}$ [89].

\section{Author Contribution Information}

All authors participated in the statement of the problem and discussion of the results. L. Gasparyan and F. Gasparyan conducted literature review. F. Gasparyan and V. Simonyan built equivalent circuits and participated in writing the text of the article.

\section{Conflict of Interest}

The authors declare no conflicts of interest regarding the publication of this paper.

\section{References}

[1] Gasparyan, L., Mazo, I., Simonyan, V. and Gasparyan, F. (2019) DNA Sequencing: Current State and Prospects of Development. Open Journal of Biophysics, 9, 169-197. https://doi.org/10.4236/ojbiphy.2019.93013

[2] Gasparyan, L., Mazo, I., Gasparyan, F. and Simonyan, V. (2020) DNA Sequencing Modified Method through Effective Regulation of Its Translocation Speed in Aqueous Solution. Open Journal of Biophysics, 10, 96-112. https://doi.org/10.4236/ojbiphy.2020.102009

[3] Gasparyan, L., Mazo, I., Simonyan, V. and Gasparyan, F. (2020) Noises and Signal-to-Noise Ratio of Nanosize EIS and ISFET Biosensors. Open Journal of Biophysics, 10, 1-12. https://doi.org/10.4236/ojbiphy.2020.101001

[4] Hoogerheide, D.P., Garaj, S. and Golovchenko, J.A. (2009) Probing Surface Charge Fluctuations with Solid-State Nanopores. Physical Review Letters, 102, 256804-256810. https://doi.org/10.1103/PhysRevLett.102.256804

[5] Pedone, D., Langecker, M., et al. (2010) Fabrication and Electrical Characterization of a Pore-Cavity-Pore Device. Journal of Physics: Condensed Matter, 22, Article ID: 454115. https://doi.org/10.1088/0953-8984/22/45/454115

[6] Powell, M.R., Vlassiouk, I., Martens, C. and Siwy, Z.S. (2009) Nonequilibrium 1/f 
Noise in Rectifying Nanopores. Physical Review Letters, 103, Article ID: 248104. https://doi.org/10.1103/PhysRevLett.103.248104

[7] Smeets, R.M.M., Dekker, N.H. and Dekker, C. (2009) Low Frequency Noise in Solid-State Nanopores. Nanotechnology, 20, Article ID: 095501.

https://doi.org/10.1088/0957-4484/20/9/095501

[8] Krems, M., Zwolak, M., et al. (2009) Effect of Noise on DNA Sequencing via Transverse Electronic Transport. Biophysical Journal, 97, 1990-1996. https://doi.org/10.1016/j.bpj.2009.06.055

[9] Lagerqvist, J., Zwolak, M. and Di Ventra, M. (2006) Fast DNA Sequencing via Transverse Electronic Transport. Nano Letters, 6, 779-782. https://doi.org/10.1021/nl0601076

[10] Zwolak, M. and Di Ventra, M. (2005) Electronic Signature of DNA Nucleotides via Transverse Transport. Nano Letters, 5, 421-424. https://doi.org/10.1021/nl048289w

[11] Lagerqvist, J., Zwolak, M. and Di Ventra, M. (2007) Influence of the Environment and Probes on Rapid DNA Sequencing via Transverse Electronic Transport. Biophysical Journal, 93, 2384-2390. https://doi.org/10.1529/biophysj.106.102269

[12] Lagerqvist, J., Zwolak, M. and Ventra, M.D. (2007) Comment on Characterization of the Tunneling Conductance across DNA Bases. Physical Review E, 76, Article ID: 013901. https://doi.org/10.1103/PhysRevE.76.013901

[13] Gasparyan, L., Mazo, I., Simonyan, V. and Gasparyan, F. (2019) ISFET Based DNA Sensor: Current-Voltage Characteristic and Sensitivity to DNA Molecules. Open Journal of Biophysics, 9, 239-253. https://doi.org/10.4236/ojbiphy.2019.94017

[14] Ventra, M.D. (2008) Electrical Transport in Nanoscale Systems. Cambridge University Press, Cambridge, UK.

[15] Lee, M.-H., Kumar, A., Park, K.-B., Cho, S.-Y., et al. (2014) A Low-Noise Solid-State Nanopore Platform Based on a Highly Insulating Substrate. Scientific Reports, 4, Article No. 7448. https://doi.org/10.1038/srep07448

[16] Fanget, A. (2013) Towards Tunneling Electrodes for Nanopore-Based DNA Sequencing. Thèse No. 5700, Ecole Polytechnique Fèdèrale de Lausanne, Suisse, 2013.

[17] Garaj, S., Hubbard, W., Reina, A., Kong, J., Branton, D. and Golovchenko, J.A. (2010) Graphene as a Subnanometre Trans-Electrode Membrane. Nature, 467, 190-193. https://doi.org/10.1038/nature09379

[18] Merchant, C.A., Healy, K., Wanunu, M., et al. (2010) DNA Translocation through Graphene Nanopores. Nano Letters, 10, 2915-2921.

https://doi.org/10.1021/nl101046t

[19] Schneider, G.F., Kowalczyk, S.W., Calado, V.E., et al. (2010) DNA Translocation through Graphene Nanopores. Nano Letters, 10, 3163-3167. https://doi.org/10.1021/nl102069z

[20] Wanunu, M., Dadosh, T., Ray, V., Jin, J., McReynolds, L. and Drndić, M. (2010) Rapid Electronic Detection of Probe-Specific microRNAs Using Thin Nanopore Sensors. Nature Nanotechnology, 5, 807-814. https://doi.org/10.1038/nnano.2010.202

[21] Morikawa, T., Yokota, K., Tanimoto, S., Tsutsui, M. and Taniguchi, M. (2017) Detecting Single-Nucleotides by Tunneling Current Measurements at Sub-MHz Temporal Resolution. Sensors (Basel), 17, 885-891. https://doi.org/10.3390/s17040885

[22] Morikawa, T., Yokota, K., Tsutsui, M. and Taniguchi, M. (2017) Fast and Low-Noise Tunneling Current Measurements for Single-Molecule Detection in an Electrolyte Solution Using Insulator-Protected Nanoelectrodes. Nanoscale, 9, 4076-4081. 
https://doi.org/10.1039/C6NR09278K

[23] Das, M.P. and Bhuyan, M. (2013)Modeling of pH Dependent Electrochemical Noise in Ion Sensitive Field Effect Transistors ISFET. Sensors and Transducers, 149, 102-108. https://www.sensorsportal.com/HTML/DIGEST/P 1134.htm

[24] Dean, M.J., Shinwari, M.W., Ranuárez, J.C. and Landheer, D. (2006) Noise Considerations in Field-Effect Biosensors. Journal of Applied Physics, 100, Article ID: 074703. https://doi.org/10.1063/1.2355542

[25] Syu, Y., Hsu, W. and Linz, C. (2018) Review-Field-Effect Transistor Biosensing: Devices and Clinical Applications. ECS Journal of Solid State Science and Technology, 7, Q3196-Q3207. https://doi.org/10.1149/2.0291807jss

[26] Bergveld, P. (1981) The Operation of an ISFET as an Electronic Device. Sensors and Actuators, 1, 17-29. https://doi.org/10.1016/0250-6874(81)80004-2

[27] Hemmerli, A., Janata, J. and Brophy, J.J. (1982) Electrochemistry of Chemically Sensitive Field Effect Transistors. Journal of the Electrochemical Society, 129, 2306-2312. https://doi.org/10.1149/1.2123500

[28] Barabash, R.P. and Cobbold, R.S.C. (1983) Basic Limitations of ISFET and Silicon Pressure Transducers: Noise Theory, Models and Device Scaling. Sensors and Actuators, 4, 427-438. https://doi.org/10.1016/0250-6874(83)85054-9

[29] Yates, D.E., Levine, S. and Healy, T.W. (1974) Site-Binding Model of the Electrical Double Layer at the Oxide/Water Interface. Journal of the Chemical Society, Faraday Transactions, 70, 1807-1818. https://doi.org/10.1039/f19747001807

[30] Abrahamian, Yu., Martirissyan, R., Gasparyan, F. and Kocharyan, K. (2004) Methods and Materials for Remote Sensing. Infrared Photo-Detectors, Radiometers and Arrays. Kluwer Academic Publishers, Boston/Dordrecht/New York/London. https://doi.org/10.1007/978-1-4419-9025-9

[31] Van der Ziel, A. (1970) Noise Sources, Characterization, Measurements. Prentice-Hall, Englewood Cliffs, NJ.

[32] Gasparyan, F.V. (2009) Photoresponse of LAPS with Different Species Membranes: Modeling and Simulation. Sensors \& Transducers Journal, 111, 141-154.

[33] Hassibi, A., Navid, R., Dutton, R.W. and Lee, T.H. (2004). Comprehensive Study of Noise Processes in Electrode Electrolyte Interfaces. Journal of Applied Physics, 96, 1074-1082. https://doi.org/10.1063/1.1755429

[34] Hassibi, A., Zahedi, S., Navid, R., Dutton, R.W. and Lee, T.H. (2005) Biological Shot-Noise and Quantum-Limited Signal-to-Noise Ratio in Affinity-Based Biosensors. Journal of Applied Physics, 97, Article ID: 084701. https://doi.org/10.1063/1.1861970

[35] Massobrio, G., Martinoa, S. and Grattarola, M. (1992) Light-Addressable Chemical Sensors: Modeling and Computer Simulations. Sensors and Actuators B: Chemical, 7, 484-487. https://doi.org/10.1016/0925-4005(92)80348-2

[36] Tyagai, V.A. (1971) Faradaic Noise of Complex Electrochemical Reactions. Electrochimica Acta, 16, 1647-1654. https://doi.org/10.1016/0013-4686(71)85075-2

[37] Johnston, I.G. (2012) The Chaos within: Exploring Noise in Cellular Biology. Significance, 19, 17-21. https://doi.org/10.1111/j.1740-9713.2012.00586.x

[38] Kaern, M., Elston, T.R., Blake, W.J. and Collins, J.J. (2005) Stochasticity in Gene Expression: From Theories to Phenotypes. Nature Reviews Genetics, 6, 451-464. https://doi.org/10.1038/nrg1615

[39] Maheshri, N. and O'Shea, E.K. (2007) Living with Noisy Genes: How Cells Function Reliably with Inherent Variability in Gene Expression. Annual Review of Biophysics 
and Biomolecular Structure, 36, 413-434.

https://doi.org/10.1146/annurev.biophys.36.040306.132705

[40] Breed, G. (2006) Noise and Spurious in Digital Systems and Digitized Signals. In: High Frequency Electronics, Summit Technical Media, LLC, 50-52. https://www.highfrequencyelectronics.com/Sep06/HFE0906 Tutorial.pdf

[41] Heerema, S.J., Schneider, G.F., Rozemuller, M., Vicarelli, L., Zandbergen, H.W. and Dekker, C. (2015) 1/f Noise in Graphene Nanopores. Nanotechnology, 26, Article ID: 074001. https://doi.org/10.1088/0957-4484/26/7/074001

[42] Chim, W.K., Leong, K.K. and Choi, W.K. (2001) Random Telegraphic Signals and Low-Frequency Noise in Rapid-Thermal-Annealed Silicon-Silicon Oxide Structures. Japanese Journal of Applied Physics, 40, 1-6. https://doi.org/10.1143/JJAP.40.1

[43] Gasparyan, F.V., Melkonyan, S.V., Aroutiounyan, V.M. and Asriyan, H.V. (2000) 1/f Noises of Homopolar and Heteropolar Semiconductors. International Journal of Modern Physics B, 14, 751-760. https://doi.org/10.1142/S0217979200000637

[44] Jakobson, C., Bloom, I. and Nemirovsky, Y. (1998) 1/f Noise in CMOS Transistors for Analog Applications from Subthreshold to Saturation. Solid-State Electronics, 42, 1807-1817. https://doi.org/10.1016/S0038-1101(98)00162-2

[45] Hooge, F.N. (1969) 1/f Noise Is No Surface Effect. Physics Letters A, 29, 139-140. https://doi.org/10.1016/0375-9601(69)90076-0

[46] Hooge, F.N., Kleinpenning, T.G.M. and Vandamme, L.K.J. (1981) Experimental Studies on 1/f Noise. Reports on Progress in Physics, 44, 479-532.

https://doi.org/10.1088/0034-4885/44/5/001

[47] Hooge, F.N. (1994) 1/f Noise Sources. IEEE Transactions on Electron Devices, 41, 1926-1935. https://doi.org/10.1109/16.333808

[48] Hung, K.K., Ko, P.K., Hu, C. and Cheng, Y.C. (1990) A Unified Model for the Flicker Noise in Metal-Oxide-Semiconductor Field-Effect Transistors. IEEE Transactions on Electron Devices, 37, 654-665. https://doi.org/10.1109/16.47770

[49] Melkonyan, S.V., Gasparyan, F.V., Aroutiounyan, V.M. and Asriyan, H.V. (1998) Temperature Chaos and the Lattice Character of the Hooge Parameter in Semiconductors. Modern Physics Letters B, 12, 1245-1254. https://doi.org/10.1142/S0217984998001475

[50] Melkonyan, S.V., Gasparyan, F.V., Aroutiounian, V.M. and Korman, C.E. (2003) Current Carrier Mobility Fluctuations in Homogeneous Semiconductors. SPIEs First International Symposium on Fluctuations and Noise, 5115, 412-420.

[51] Melkonyan, S.V., Gasparyan, F.V., Aroutiounian, V.M. and Asriyan, H.V. (2005). 1/f-Type Noise in View of Phonons Interface Percolation Dynamics. AIP Conference Proceedings, 780, 87-91. https://doi.org/10.1063/1.2036705

[52] Melkonyan, S.V., Aroutiounian, V.M., Gasparyan, F.V. and Korman, C.E. (2005) Peculiarities of Electron Distribution Function's Fluctuations Damping in Homogeneous Semiconductors. Physica B: Condensed Matter, 357, 398-407. https://doi.org/10.1016/j.physb.2004.12.004

[53] Melkonyan, S.V., Aroutiounian, V.M., Gasparyan, F.V. and Asriyan, H.V. (2006) Phonon Mechanism of Mobility Equilibrium Fluctuation and Properties of 1/f-Noise. Physica B: Condensed Matter, 382, 65-70. https://doi.org/10.1016/j.physb.2006.01.521

[54] Melkonyan, S.V., Gasparyan, F.V. and Asriyan, H.V. (2007) Main Sources of Electron Mobility Fluctuations in Semiconductors. SPIE Fourth International Sympo- 
sium on Fluctuations and Noise, 6600, 66001K.

[55] Peransin, J.-M., Vignaud, P., Rigaud, D. and Vandamme, L.K.J. (1990) 1/f Noise in MODFETs at Low Drain Bias. IEEE Transactions on Electron Devices, 37, 2250-2253. https://doi.org/10.1109/16.59916

[56] McWhorter, A.L. (1957) 1/f Noise and Germanium Surface Properties. In: Kingston, R.H., Ed., Semiconductor Surface Physics, University of Pennsylvania Press, Philadelphia, 207-228.

[57] Hooge, F.N. (2004) 1/f Noise Sources. In: Sikula, J. and Levinshtein, M., Eds., Advanced Experimental Methods for Noise Research in Nanoscale Electronic Devices. NATO Science Series II: Mathematics, Physics and Chemistry (II. Mathematics, Physics and Chemistry), Vol. 151, 3-10. https://doi.org/10.1007/1-4020-2170-4 1

[58] Männik, J., Heller, I., Janssens, A.M., Lemay, S.G. and Dekker, C. (2008) Charge Noise in Liquid-Gated Single-Wall Carbon Nanotube Transistors. Nano Letters, 8 , 685-688. https://doi.org/10.1021/nl073271h

[59] Gasparyan, F.V., Vitusevich, S.A., Offenhäusser, A. and Schöning, M.J. (2011) Modified Charge Fluctuation Noise Model for Electrolyte-Insulator-Semiconductor Devices. Modern Physics Letters B, 25, 831-840. https://doi.org/10.1142/S0217984911026103

[60] Hooge, F.N. (1976) Discussion of Recent Experiments on 1/f Noise. Physica, 60, 130-144. https://doi.org/10.1016/0031-8914(72)90226-1

[61] Vandamme, L.K.J. and de Werd, H.M.M. (1980) 1/f Noise Model for MOSTs Biased in the Nonohmic Region. Solid-State Electronics, 23, 325-329.

https://doi.org/10.1016/0038-1101(80)90199-9

[62] Hooge, F.N. and Vandamme, L.K.J. (1978) Lattice Scattering Causes 1/f Noise. Physics Letters A, 66, 315-316. https://doi.org/10.1016/0375-9601(78)90249-9

[63] Gasparyan, F.V., Poghossian, A., Vitusevich, S.A., et al. (2009) 1/f-Noise in EIS Bio-Sensors Functionalized with 3 Layer-by-Layer PAMAM/Single Walled Carbon Nanotubes. Proceedings of the 20 th International Conference on Noise and Fluctuation, Pisa, Italy, 14-19 June 2009, 133-136.

[64] Gasparyan, F.V., Poghossian, A., Vitusevich, S.A., et al. (2011) Low-Frequency Noise in Field-Effect Devices Functionalized with Dendrimer/Carbon-Nanotube Multilayers. IEEE Sensors Journal, 11, 142-149. https://doi.org/10.1109/ISEN.2010.2052355

[65] Christensson, S., Lundstrom, I. and Svensson, C. (1968) Low Frequency Noise in MOS Transistors-I. Theory. Solid-State Electronics, 11, 797-812. https://doi.org/10.1016/0038-1101(68)90100-7

[66] Christensson, S. and Lundstrom, I. (1968) Low Frequency Noise in MOS Transistors-II. Experiments. Solid-State Electronics, 11, 813-820. https://doi.org/10.1016/0038-1101(68)90101-9

[67] Reimbold, G. (1984) Modified 1/f Trapping Noise and Experiments in MOS Transistors Biased from Weak to Strong Inversion-Influence of Interface States. IEEE Transactions on Electron Devices, 31, 1190-1198. https://doi.org/10.1109/T-ED.1984.21687

[68] Vandamme, L.K.J., Li, X. and Rigaud, D.M. (1994) 1/f Noise in MOS Devices, Mobility or Number Fluctuations? IEEE Transactions on Electron Devices, 41, 1936-1945. https://doi.org/10.1109/16.333809

[69] Jacobson, C.G. and Nemirovsky, Y. (1999) 1/f Noise in Ion Sensitive Field Effect Transistor from Subthreshold to Saturation. IEEE Transactions on Electron Devic- 
es, 46, 259-261. https://doi.org/10.1109/16.737468

[70] Kaisti, M. (2017) Detection Principles of Biological and Chemical FET Sensors. Biosensors and Bioelectronics, 98, 437-448.

https://doi.org/10.1016/j.bios.2017.07.010

[71] Zagni, N., Pavan, P. and Alam, M.A. (2019) Two-Dimensional MoS 2 Negative Capacitor Transistors for Enhanced (Super Nernstian) Signal-to-Noise Performance of Next-Generation Nano Biosensors. Applied Physics Letters, 114, Article ID: 233102. https://doi.org/10.1063/1.5097828

[72] McAndrew, C.C., Coram, G., Blaum, A. and Pilloud, O. (2005) Correlated Noise Modeling and Simulation. NSTI-Nanotech 2005, 40-45.

https://www.semanticscholar.org/paper/Correlated-Noise-Modeling-and-Simulatio n-McAndrew-Coram/579c166296242367f12f5b98071c9b515f7fc9ad

[73] Brederlow, R., Weber, W., et al. (1998) A Physically Based Model for Low-Frequency Noise of Poly-Silicon Resistors. International Electron Devices Meeting 1998, 6-9 December 1998, San Francisco, CA, 89-92.

https://doi.org/10.1109/IEDM.1998.746286

[74] Tsividis. Y. (1999) Operation and Modeling of the MOS Transistor. 2nd Edition, McGraw-Hill, New York.

[75] Taur, Y. and Ning, T.H. (2009) Fundamentals of Modern VLSI Devices. 2nd Edition, Cambridge University Press, Cambridge.

https://doi.org/10.1017/CBO9781139195065

[76] Meixner, L.K. and Koch, S. (1992) Simulation of ISFET Operation Based on the Site-Binding Model. Sensors and Actuators B: Chemical, 6, 315-318. https://doi.org/10.1016/0925-4005(92)80077-B

[77] Jin, B., Lee, G.-Y., Park, C., Kim, D., Choi, W., Yoo, J.-W., Pyun, J.-C. and Lee, J.-S. (2018) Electrical Characteristics and $\mathrm{pH}$ Response of a Parylene-H Sensing Membrane in a Si-Nanonet Ion-Sensitive Field-Effect Transistor. Sensors, 18, 3892. https://doi.org/10.3390/s18113892

[78] Haartman, M.V. and Ostling, M. (2007) Low-Frequency Noise in Advanced MOS Devices. Springer, Berlin/Heidelberg, Germany, 216. https://doi.org/10.1007/978-1-4020-5910-0

[79] Gasparyan, F. (2010) Excess Noises in (Bio-)Chemical Nanoscale Sensors. Sensors \& Transducers Journal, 122, 72-84. https://www.sensorsportal.com/HTML/DIGEST/november_2010/P 711.pdf

[80] Gasparyan, F.V. (2013) Chapter 11: Noise Reduction in (Bio-)Chemical Sensors Functionalized with Carbon Nanotube Multilayers. In: Vaseashta, A. and Khudaverdyan, S., Eds., Advanced Sensors for Safety and Security, NATO Science for Peace and Security Series B: Physics and Biophysics, Springer, Dordrecht, 139-150. https://doi.org/10.1007/978-94-007-7003-4_11

[81] Gasparyan, F., Zadorozhnyi, I., Khondkaryan, H., Arakelyan, A. and Vitusevich, S. (2018) Photoconductivity, pH-Sensitivity, Noise, and Channel Length Effects in Si Nanowire FET Sensors. Nanoscale Research Letters, 13, 87-96. https://doi.org/10.1186/s11671-018-2494-5

[82] Vitusevich, S. and Gasparyan, F. (2011) Chapter 11: Low-Frequency Noise Spectroscopy at Nanoscale: Carbon Nanotube Materials and Devices. In: Marulanda, J.M., Ed., Carbon Nanotubes Applications on Electron Devices, IntechOpen Limited, London, 257-296. https://doi.org/10.5772/20026

[83] Gasparyan, F.V., Asriyan, H.V., Melkonyan, S.V. and Korman, C.E. (2010) Method 
of 1/f Noise Reduction and Noise Level Manipulation in Semiconductor Based Devices. U.S. Patent Application for Letters Patent of the United States No. 61/332, 408.

[84] Pavelka, J., Sikula, J., et al. (2002) Noise and Transport Characterization of Tantalum Capacitors. Microelectronics Reliability, 42, 841-847.

https://doi.org/10.1016/S0026-2714(02)00013-6

[85] Zhigal'skii, G.P., Putrya, M.G. and Fedorov, A.S. (1989) Effect of Silicon-Wafer Preoxidation on the Low-Frequency Noise of MIS Structures. Radiophysics and Quantum Electronics, 32, 862-868. https://doi.org/10.1007/BF01038815

[86] Chertouk, M. and Chovet, A. (1996) Origins and Characterization of Low-Frequency Noise in GaAs MESFET's Grown on InP Substrates. IEEE Transactions on Electron Devices, 43, 123-129. https://doi.org/10.1109/16.477602

[87] Haartman, M.V. and Ostling, M. (2007) Effect of Channel Positioning on the 1/f Noise in Silicon-Oninsulator Metal-Oxide-Semiconductor Field-Effect Transistors. Journal of Applied Physics, 101, Article ID: 03456. https://doi.org/10.1063/1.2433772

[88] Tian, H. and Gamal, A.E. (2001) Analysis of 1/f Noise in Switched MOSFET Circuits. IEEE Transactions on Circuits and Systems II: Analog and Digital Signal Processing, 48, 151-157. https://doi.org/10.1109/82.917783

[89] Jomaah, J., Balestra, F. and Ghibaudo, G. (2005) Low Frequency Noise in Advanced Si Bulk and SOI MOSFETs. Journal of Telecommunications and Information Technology, 1, 24-33. 